\title{
Gravitational Lorentz force and the description of the gravitational interaction
}

\author{
V. C. de Andrade and J. G. Pereira \\ Instituto de Física Teórica, Universidade Estadual Paulista, Rua Pamplona 145, 01405-900 São Paulo, Brazil
}

(Received 21 March 1997)

\begin{abstract}
In the context of a gauge theory for the translation group, we have obtained, for a spinless particle, a gravitational analogue of the Lorentz force. Then, we have shown that this force equation can be rewritten in terms of magnitudes related to either the teleparallel or the Riemannian structures induced in spacetime by the presence of the gravitational field. In the first case, it gives a force equation, with torsion playing the role of force. In the second, it gives the usual geodesic equation of general relativity. The main conclusion is that scalar matter is able to feel any one of the above spacetime geometries, the teleparallel and the metric ones. Furthermore, both descriptions are found to be completely equivalent in the sense that they give the same physical trajectory for a spinless particle in a gravitational field. [S0556-2821(97)05620-8]

PACS number(s): 04.20.Cv, 04.50.+h
\end{abstract}

\section{INTRODUCTION}

The notion of absolute parallelism (or teleparallelism) was introduced by Einstein in the 1920s in his attempt to unify gravitation and electromagnetism. About three decades later, works by Møller [1], Pellegrini and Plebanski [2], and Hayashi and Nakano [3] produced a revival of those ideas, which since then have received considerable attention, mainly in the context of gauge theories for the Poincare and the translation groups [4-10].

The scene of the teleparallel theories of gravitation is the Weitzenböck spacetime [11], a space presenting torsion, but no curvature. The teleparallel description of gravitation is believed to be equivalent, at least macroscopically, to the general relativity description, whose stage set is provided by a Riemann spacetime, a space presenting curvature, but no torsion. If this equivalence is in fact true and effective, the gravitational interaction might have two equivalent descriptions, one of them in terms of torsion only and another one in terms of curvature only.

With the purpose of exploring this equivalence, we study in this paper a gauge theory for the translation group, trying to stay as close as possible to the usual scheme of the gauge models for internal groups. This means essentially that we start by considering spacetime to be a Minkowski space. The resulting model, as we are going to see, will be quite analogous to the $\mathrm{U}(1)$ electromagnetic gauge theory. Relying on this analogy and considering the motion of a spinless test particle in a translational gauge gravitational field, we deduce the gravitational analogue of the Lorentz force equation. This equation describes the trajectory of the particle submitted to a gauge gravitational field in a flat spacetime. Now, as a result of the spacetime character of translations, the corresponding gauge theory will differ from the usual gauge models in many ways, the most significant being the presence of a tetrad field. A tetrad field defines in a natural way a linear Cartan connection with respect to which the tetrad is parallel. For this reason, tetrad theories have received the name of teleparallelism, or absolute parallelism. A tetrad field defines also in a natural way a Riemannian metric, in terms of which a Levi-Cività connection can be de- fined. On the other hand, as is well known, torsion and curvature are properties of a connection [12], and many different connections can be defined on the same space. Therefore, in the specific case of a tetrad theory, we can say that the presence of a nontrivial tetrad field in the gauge theory induces both, a teleparallel and a Riemannian structure in spacetime. The first is related to the Cartan connection, a connection presenting torsion, but no curvature. The second is related to the Levi-Cività connection, a connection presenting curvature, but no torsion. Then, owing to the universality of the gravitational interaction, it turns out to be possible to link these geometrical structures with gravitation. However, despite the simultaneous presence of these two geometrical structures, we will show in this paper that, in agreement with the equivalence alluded to above, the description of the gravitational interaction requires only one of the above structures. In other words, the gravitational interaction can be described alternatively in terms of magnitudes related to the teleparallel or to the Riemannian structures induced in spacetime by the nontrivial tetrad field. Concerning the dynamics of the gravitational field, it has already been shown [13] that this is in fact the case: The HilbertEinstein Lagrangian of general relativity, linear in the scalar curvature, is completely equivalent to the Lagrangian of a translational gauge theory, quadratic in the torsion tensor. Similarly, we will show that the gravitational Lorentz force equation, which describes the motion of a particle submitted to a gauge gravitational field in a flat spacetime, can be rewritten in terms of magnitudes related to either the teleparallel or the Riemannian geometry of spacetime. In the first case, the resulting equation is not a geodesic but a force equation, which means that the trajectories followed by scalar matter are not geodesics of the induced Weitzenböck spacetime. In the second case, the gravitational Lorentz force becomes the geodesic equation of general relativity, which means that the trajectories followed by scalar matter are geodesics of the induced Riemann spacetime. As both descriptions are obtained from the same force equation, we conclude that they are completely equivalent, which means essentially that scalar matter is able to feel any one of the above spacetime geometries. 


\section{GAUGE THEORY FOR THE TRANSLATION GROUP}

We start by assuming spacetime to be a Minkowski space. The second half of the greek alphabet $(\mu, \nu, \rho, \ldots=1,2,3,4)$ will be used to denote indices related to this space. Its coordinates, therefore, will be denoted by $x^{\mu}$ and its metric by $\eta_{\mu \nu}$. At each point of spacetime, there is a tangent space attached to it, given also by a Minkowski space, which will be the fiber of the corresponding tangent bundle. We use the first half of the greek alphabet $(\alpha, \beta, \gamma, \ldots=1,2,3,4)$ to denote indices related to this space. Its coordinates, therefore, will be denoted by $x^{\alpha}$ and its metric by $\eta_{\alpha \beta}$. As gauge transformations take place in this space, these will also be the algebra indices of the gauge model. The holonomic derivatives in these two spaces can be identified by

$$
\partial_{\mu}=\left(\partial_{\mu} x^{\alpha}\right) \partial_{\alpha}, \quad \partial_{\alpha}=\left(\partial_{\alpha} x^{\mu}\right) \partial_{\mu},
$$

where $\partial_{\mu} x^{\alpha}$ is a trivial holonomic tetrad, with $\partial_{\alpha} x^{\mu}$ its inverse.

A gauge transformation is defined as a local translation of the fiber coordinates,

$$
x^{\prime \alpha}=x^{\alpha}+a^{\alpha}\left(x^{\mu}\right),
$$

with $a^{\alpha}\left(x^{\mu}\right)$ the corresponding parameters. It can be written in the form $x^{\prime}=U x$, where $U$ is an element of the translation group. For an infinitesimal transformation,

$$
U=1+\delta a^{\alpha} P_{\alpha},
$$

with $\delta a^{\alpha}$ representing the infinitesimal parameters and $P_{\alpha}=\partial_{\alpha}$ standing for the generators of infinitesimal translations, which satisfy

$$
\left[P_{\alpha}, P_{\beta}\right]=0 \text {. }
$$

In terms of these generators, the infinitesimal version of transformation (2) becomes

$$
\delta x^{\alpha}=\delta a^{\beta} P_{\beta} x^{\alpha} .
$$

Let us consider now a general source field $\Phi\left(x^{\mu}\right)$. Its gauge transformation does not depend on the spin character and is given by

$$
\Phi^{\prime}\left(x^{\mu}\right)=U \Phi\left(x^{\mu}\right) .
$$

The corresponding infinitesimal transformation, therefore, is

$$
\delta \Phi=\delta a^{\alpha} P_{\alpha} \Phi
$$

with $\delta \Phi$ standing for the functional change at the same $x^{\mu}$, which is the relevant transformation for gauge theories. It is important to remark that the translation generators are able to act on any source field through their arguments because of identification (1).

In order to define the gauge-covariant derivative of $\Phi\left(x^{\mu}\right)$, we must first introduce the gauge potentials of the model, which will be denoted by

$$
B_{\mu}=B^{\alpha}{ }_{\mu} P_{\alpha} .
$$

Using the general definition of gauge covariant derivatives,

$$
D_{\mu}=\partial_{\mu}+c^{-2} B^{\alpha}{ }_{\mu} \frac{\delta}{\delta a^{\alpha}},
$$

where the velocity of light $c$ was introduced for dimensional reasons, the covariant derivative of $\Phi\left(x^{\mu}\right)$ turns out to be

$$
D_{\mu} \Phi=\partial_{\mu} \Phi+c^{-2} B_{\mu}^{\alpha} P_{\alpha} \Phi \text {. }
$$

As the generators are derivatives which act on the fields through their arguments, every source field in nature will respond to their action and consequently will couple to the gauge potentials. In other words, every source field in nature will feel gravitation the same way. This is the origin of the concept of universality according to this model.

From the covariance requirement for $D_{\mu}$, we can get the gauge transformation of the potentials:

$$
B_{\mu}^{\prime}=U B_{\mu} U^{-1}+c^{2} U \partial_{\mu} U^{-1}
$$

The corresponding infinitesimal transformation is

$$
B_{\mu}^{\prime \alpha}=B_{\mu}^{\alpha}-c^{2} \partial_{\mu} \delta a^{\alpha} .
$$

The field strength $F^{\alpha}{ }_{\mu \nu}$ is defined as the covariant derivative of the gauge potential $B^{\alpha}{ }_{\mu}$, and analogously to the $\mathrm{U}(1)$ electromagnetic gauge theory, it reads

$$
F_{\mu \nu}^{\alpha}=\partial_{\mu} B_{\nu}^{\alpha}-\partial_{\nu} B_{\mu}^{\alpha} .
$$

Notice that, as expected for an Abelian theory, $F_{\mu \nu}^{\alpha}$ is invariant under a gauge transformation. Consequently, its ordinary and gauge-covariant derivatives coincide.

The dynamics of the gauge fields, as usual, can be obtained from a Lagrangian quadratic in the field strength,

$$
\mathcal{L}=\frac{1}{16 \pi G}\left[\frac{1}{4} F^{\alpha}{ }_{\mu \nu} F^{\beta}{ }_{\theta \rho} \eta^{\mu \theta} N_{\alpha \beta}{ }^{\nu \rho}\right],
$$

where $G$ is the gravitational constant and

$$
N_{\alpha \beta}^{\nu \rho}=\eta_{\alpha \beta} \eta^{\nu \rho}
$$

\section{GRAVITATIONAL LORENTZ FORCE}

Let us now consider the motion of a particle of mass $m$ in a gravitational field described by a translation gauge theory. As spacetime is a Minkowski space, we can rely on an analogy with the electromagnetic case to obtain the corresponding gravitational interaction. Thus, analogously to what occurs in electrodynamics [14], we assume the interaction of the particle with the gravitational field to be described by the action

$$
c^{-2} \int_{a}^{b} B^{\alpha}{ }_{\mu} p_{\alpha} d x^{\mu}
$$

with the integration taken along the world line of the particle. In this expression, $p_{\alpha}$ is the Noether conserved charge under the transformations of the gauge group $[15,16]$. In other words, $p_{\alpha}$ is the four-momentum,

$$
p_{\alpha}=m c u_{\alpha},
$$


of the particle, with $u_{\alpha}$ its four-velocity. Therefore, the complete action for a particle in a gravitational field is given by

$$
S=\int_{a}^{b} L d s \equiv \int_{a}^{b}\left[-m c \sqrt{-u^{2}}+\frac{m}{c} B^{\alpha}{ }_{\mu} u_{\alpha} u^{\mu}\right] d s,
$$

with $d s=\left(\eta_{\mu \nu} d x^{\mu} d x^{\nu}\right)^{1 / 2}$ the Minkowski interval and $u^{2}=\eta_{\mu \nu} u^{\mu} u^{\nu}$. Notice that, in writing this action, we have already assumed the equality between inertial and gravitational masses, as stated by the (weak) equivalence principle [17].

Next, we make use of the Euler-Lagrange equation to obtain the equations of motion. The result is

$$
m c\left[\frac{d u_{\mu}}{d s}+c^{-2} B^{\alpha}{ }_{\mu} \frac{d u_{\alpha}}{d s}\right]=\frac{m}{c} F^{\alpha}{ }_{\mu \nu} u_{\alpha} u^{\nu} .
$$

Using the relation

$$
\frac{d u_{\mu}}{d s}=\left(\frac{\partial x^{\alpha}}{\partial x^{\mu}}\right) \frac{d u_{\alpha}}{d s}
$$

we get finally

$$
\left(\partial_{\mu} x^{\alpha}+c^{-2} B_{\mu}^{\alpha}\right) \frac{d u_{\alpha}}{d s}=c^{-2} F_{\mu \nu}^{\alpha} u_{\alpha} u^{\nu} .
$$

This is the gravitational analogue of the Lorentz force. Its solution determines the trajectory of the particle in a flat spacetime. It is interesting to notice that, while in the electromagnetic case the particle four-acceleration is proportional to $e / m$, with $e$ its electric charge, in the gravitational case the mass disappears from the equation of motion. This is, of course, a consequence of the assumed equivalence between the gravitational and inertial masses.

\section{INDUCED SPACETIME GEOMETRY}

Up to this point, spacetime has been considered to be the Minkowski space. However, as we are going to see, the presence of a nontrivial tetrad field in the translation gauge theory induces further structures in spacetime. Owing to the universality of the gravitational interaction, it will then be possible to relate these structures to the presence of gravitation.

By using Eq. (1), the covariant derivative (10) of a general source field can be rewritten in the form

$$
D_{\mu} \Phi=h^{\alpha}{ }_{\mu} \partial_{\alpha} \Phi
$$

where

$$
h^{\alpha}{ }_{\mu}=\partial_{\mu} x^{\alpha}+c^{-2} B^{\alpha}{ }_{\mu} \equiv D_{\mu} x^{\alpha}
$$

is a tetrad field. Notice that the gravitational field appears as the nontrivial part of the tetrad [18]. Making use of Eqs. (5) and (12), it is easy to see that, as in fact it should be, the tetrad is gauge invariant:

$$
h^{\prime \alpha}{ }_{\mu}=h^{\alpha}{ }_{\mu} .
$$

Its gauge-covariant derivative, therefore, turns out to be the same as the ordinary derivative.
The expression for the gauge-covariant derivative operator of source fields,

$$
D_{\mu}=h^{\alpha}{ }_{\mu} \partial_{\alpha},
$$

is actually the definition of a nonholonomous basis. In the absence of gravitation, $h^{\alpha}{ }_{\mu}$ becomes trivial, and it reduces to the coordinate basis $\partial_{\mu}$ appearing in Eq. (1). This new basis, induced by the presence of the gravitational field, satisfies the commutation relation

$$
\left[D_{\mu}, D_{\nu}\right]=c^{-2} F^{\alpha}{ }_{\mu \nu} P_{\alpha} .
$$

Therefore, as usual in gauge theories, the commutator of covariant derivative operators yields the field strength $F^{\alpha}{ }_{\mu \nu}$. There is a difference, though: In contrast to the usual gauge theories, the field strength here will be directly related to the spacetime geometry.

In fact, as a result of the presence of a tetrad field, there always exists a naturally defined linear Cartan connection [12]

$$
\Gamma_{\mu \nu}^{\rho}=h_{\alpha}{ }^{\rho} \partial_{\nu} h_{\mu}^{\alpha},
$$

which is a connection presenting torsion, but no curvature. As a consequence, the field strength $F^{\alpha}{ }_{\mu \nu}$ can be written in the form

$$
F_{\mu \nu}^{\alpha}=c^{2} h^{\alpha}{ }_{\rho} T_{\mu \nu}^{\rho},
$$

where

$$
T_{\mu \nu}^{\rho}=\Gamma_{\nu \mu}^{\rho}-\Gamma_{\mu \nu}^{\rho}
$$

is the torsion induced in spacetime by the presence of the gravitational field. Moreover, the commutation relation (23) acquires the form

$$
\left[D_{\mu}, D_{\nu}\right]=T^{\rho}{ }_{\mu \nu} D_{\rho},
$$

indicating that torsion plays also the role of the nonholonomy of the gauge-covariant derivative.

The presence of a Cartan connection allows the introduction of a spacetime-covariant derivative which, acting for example on a spacetime covariant vector $V_{\mu}$, reads

$$
\nabla_{\nu} V_{\mu}=\partial_{\nu} V_{\mu}-\Gamma_{\mu \nu}^{\theta} V_{\theta} .
$$

From this definition, one can easily see that, as a consequence of Eq. (24),

$$
\nabla_{\nu} h_{\mu}^{\alpha}=\partial_{\nu} h_{\mu}^{\alpha}-h_{\rho}^{\alpha} \Gamma_{\mu \nu}^{\rho} \equiv 0 .
$$

This is the condition of absolute parallelism, which implies that the spacetime underlying a translational gauge theory is naturally endowed with a teleparallel structure. In other words, a Weitzenböck's four-dimensional manifold is always present when considering a gauge theory for the translation group [4].

Besides the teleparallel structure, the presence of a nontrivial tetrad induces also a Riemannian structure in spacetime. As the tetrad satisfies

$$
h^{\alpha}{ }_{\mu} h_{\alpha}{ }^{\nu}=\delta_{\mu}{ }^{\nu}, \quad h^{\alpha}{ }_{\mu} h_{\beta}{ }^{\mu}=\delta^{\alpha}{ }_{\beta},
$$


if algebra indices are raised and lowered with the Lorentzian metric $\eta^{\alpha \beta}$, tensor indices will necessarily be raised and lowered with the Riemannian metric

$$
g_{\mu \nu}=\eta_{\alpha \beta} h_{\mu}^{\alpha} h_{\nu}^{\beta} .
$$

Accordingly, a linear metric connection can be introduced:

$$
\stackrel{\circ}{\Gamma}_{\mu \nu}^{\theta}=\frac{1}{2} g^{\theta \rho}\left[\partial_{\mu} g_{\rho \nu}+\partial_{\nu} g_{\rho \mu}-\partial_{\rho} g_{\mu \nu}\right],
$$

which is a connection presenting curvature, but no torsion. Its curvature

$$
\stackrel{\circ}{R}_{\rho \mu \nu}^{\theta}=\partial_{\mu} \stackrel{\circ}{\Gamma}_{\rho \nu}^{\theta}+\stackrel{\circ}{\Gamma}_{\sigma \mu}^{\theta} \stackrel{\circ}{\Gamma}_{\rho \nu}^{\sigma}-(\mu \leftrightarrow \nu)
$$

represents the curvature induced in spacetime by the presence of the gravitational field. The connection $\stackrel{\circ}{\Gamma}_{\mu \nu}^{\theta}$, actually the Levi-Cività connection of $g_{\mu \nu}$, allows the introduction of another spacetime-covariant derivative which, acting for example on a spacetime covariant vector $V_{\mu}$, reads

$$
\stackrel{\circ}{\nabla}_{\nu} V_{\mu}=\partial_{\nu} V_{\mu}-\stackrel{\circ}{\Gamma}_{\mu \nu}^{\theta} V_{\theta} .
$$

As can be easily verified, both connections $\Gamma_{\mu \nu}^{\theta}$ and $\stackrel{\circ}{\Gamma}_{\mu \nu}^{\theta}$ preserve the metric:

$$
\stackrel{\circ}{\nabla}_{\nu} g_{\rho \mu}=\nabla_{\nu} g_{\rho \mu}=0 .
$$

Substituting now $g_{\mu \nu}$ into $\stackrel{\circ}{\Gamma}_{\mu \nu}^{\theta}$, we obtain

$$
\Gamma_{\mu \nu}^{\theta}=\stackrel{\circ}{\Gamma}_{\mu \nu}^{\theta}+K_{\mu \nu}^{\theta}
$$

where

$$
K_{\mu \nu}^{\theta}=\frac{1}{2}\left[T_{\mu}^{\theta}{ }_{\nu}+T_{\nu \mu}^{\theta}-T_{\mu \nu}^{\theta}\right]
$$

is the contorsion tensor. Notice that the curvature of the Cartan connection vanishes identically:

$$
R_{\rho \mu \nu}^{\theta}=\partial_{\mu} \Gamma_{\rho \nu}^{\theta}+\Gamma_{\sigma \mu}^{\theta} \Gamma_{\rho \nu}^{\sigma}-(\mu \leftrightarrow \nu) \equiv 0 .
$$

Substituting $\Gamma_{\mu \nu}^{\theta}$ from Eq. (35), we get

$$
R_{\rho \mu \nu}^{\theta}=\stackrel{\circ}{R}_{\rho \mu \nu}^{\theta}+(D K)_{\rho \mu \nu}^{\theta}-K_{\sigma \mu}^{\theta} K_{\rho \nu}^{\sigma}+K_{\sigma \nu}^{\theta} K_{\rho \mu}^{\sigma} \equiv 0,
$$

where

$$
(D K)_{\rho \mu \nu}^{\theta}=\partial_{\mu} K_{\rho \nu}^{\theta}+\Gamma_{\sigma \mu}^{\theta} K_{\rho \nu}^{\sigma}+\Gamma_{\rho \nu}^{\sigma} K_{\sigma \mu}^{\theta}-(\mu \leftrightarrow \nu) .
$$

From these considerations, we conclude that the presence of a nontrivial tetrad field in the translational gauge theory induces both a teleparallel and a Riemannian structure in spacetime. These structures together completely characterize the induced spacetime geometry. Moreover, we see from Eq. (38) that the Riemann curvature tensor $\stackrel{\circ}{R}_{\rho \mu \nu}^{\theta}$ induced in spacetime is such that it compensates exactly the contribution to the curvature coming from the teleparallel structure, yielding an identically zero total curvature tensor. As we are going to see, however, the description of the gravitational interaction requires only one of the above structures. In other words, the gravitational interaction can be described alternatively in terms of magnitudes related to the teleparallel or to the Riemannian geometry.

\section{DYNAMICS OF THE GAUGE FIELDS}

In the induced spacetime, the gauge field Lagrangian (14) is written as

$$
\mathcal{L}=\frac{h}{16 \pi G}\left[\frac{1}{4} F^{\alpha}{ }_{\mu \nu} F^{\beta}{ }_{\theta \rho} g^{\mu \theta} N_{\alpha \beta}{ }^{\nu \rho}\right],
$$

where $h=\operatorname{det}\left(h^{\alpha}{ }_{\mu}\right)$ is the Jacobian of the transformation (22). Because of the presence of the tetrad field, however, algebra and spacetime indices can now be changed into each other and consequently appear mixed up in the Lagrangian. This means that

$$
N_{\alpha \beta}{ }^{\nu \rho}=\eta_{\alpha \beta} g^{\nu \rho} \equiv \eta_{\alpha \beta} h_{\gamma}{ }^{\nu} h^{\gamma \rho}
$$

must now include all cyclic permutations of $\alpha, \beta$, and $\gamma$. A simple calculation shows that

$$
N_{\alpha \beta}^{\nu \rho}=\eta_{\alpha \beta} h_{\gamma}{ }^{\nu} h^{\gamma \rho}+2 h_{\alpha}{ }^{\rho} h_{\beta}{ }^{\nu}-4 h_{\alpha}{ }^{\nu} h_{\beta}{ }^{\rho} .
$$

Substituting in Eq. (40), the gauge field Lagrangian turns out to be

$$
\begin{aligned}
\mathcal{L}= & \frac{h}{16 \pi G} F^{\alpha}{ }_{\mu \nu} F^{\beta}{ }_{\theta \rho} g^{\mu \theta}\left[\frac{1}{4} h_{\delta}{ }^{\nu} h^{\delta \rho} \eta_{\alpha \beta}\right. \\
& \left.+\frac{1}{2} h_{\alpha}{ }^{\rho} h_{\beta}{ }^{\nu}-h_{\alpha}{ }^{\nu} h_{\beta}{ }^{\rho}\right] .
\end{aligned}
$$

Then, by using Eq. (25), it becomes

$$
\mathcal{L}=\frac{h c^{4}}{16 \pi G}\left[\frac{1}{4} T_{\mu \nu}^{\rho} T_{\rho}{ }^{\mu \nu}+\frac{1}{2} T_{\mu \nu}^{\rho} T_{\rho}^{\nu \mu}-T_{\rho \mu}{ }^{\rho} T^{\nu \mu}{ }_{\nu}\right] .
$$

In order to obtain the vacuum field equations, it is convenient to rewrite it as [13]

$$
\mathcal{L}=\frac{h c^{4}}{16 \pi G} S_{\rho}^{\mu \nu} T^{\rho}{ }_{\mu \nu},
$$

where

$$
S_{\rho}^{\mu \nu}=\frac{1}{4}\left(T_{\rho}^{\mu \nu}+T_{\rho}^{\mu}{ }^{\nu}-T_{\rho}^{\nu}{ }^{\mu}\right)-\frac{1}{2}\left(\delta_{\rho}{ }^{\nu} T_{\theta}{ }^{\mu \theta}-\delta_{\rho}{ }^{\mu} T_{\theta}{ }^{\nu \theta}\right) .
$$

By performing variations in $\mathcal{L}$ with respect to $B^{\alpha}{ }_{\mu}$, we get the equation

$$
\partial_{\nu} S_{\rho}^{\mu \nu}-\frac{4 \pi G}{c^{4}} t_{\rho}{ }^{\mu}=0,
$$

where

$$
\begin{aligned}
t_{\rho}{ }^{\mu}= & \frac{c^{4}}{16 \pi G}\left[4 S_{\rho}^{\mu \theta} \Gamma_{\nu \theta}^{\nu}+2 S^{\mu \nu \theta} T_{\rho \nu \theta}+T_{\sigma}{ }^{\theta \mu} T_{\theta \rho}^{\sigma}\right. \\
& \left.+T^{\theta}{ }_{\sigma}^{\mu} T_{\theta \rho}^{\sigma}-2 T_{\theta}^{\theta \mu} T_{\rho \theta}^{\theta}+\frac{1}{2} T^{\mu \theta}{ }_{\sigma} T_{\rho \theta}{ }^{\sigma}\right]-\delta_{\rho}{ }^{\mu} h^{-1} \mathcal{L}
\end{aligned}
$$

is the gauge field self-current, which in this case is the energy-momentum (pseudo)tensor of the gravitational field. 
Notice that, despite being an Abelian gauge theory, this equation presents a nonvanishing self-current which makes it quite similar to the Yang-Mills equations. However, in the present case, the self-current has a different nature as, in contrast to the standard Yang-Mills theories [19], it cannot be written as a commutator since the gauge group here is Abelian.

On the other hand, as we have already seen, the presence of a nontrivial tetrad field in the gauge theory induces also a Riemannian structure in spacetime. We consider then, in the induced Riemann spacetime, the Hilbert-Einstein Lagrangian of general relativity:

$$
\mathcal{L}=\frac{c^{4} h}{16 \pi G} \stackrel{\circ}{R} .
$$

Substituting $\stackrel{\circ}{R}$ as obtained from Eq. (38), it can be rewritten in terms of the Cartan connection only. Up to divergences, we get

$$
\mathcal{L}=\frac{h c^{4}}{16 \pi G}\left[\frac{1}{4} T^{\rho}{ }_{\mu \nu} T_{\rho}{ }^{\mu \nu}+\frac{1}{2} T^{\rho}{ }_{\mu \nu} T_{\rho}^{\nu \mu}-T_{\rho \mu}{ }^{\rho} T^{\nu \mu}{ }_{\nu}\right],
$$

which is exactly the Lagrangian (43) of the translational gauge theory. We have in this way recovered the well-known result [13] which says that the translational gauge theory, with a Lagrangian quadratic in the torsion field, is completely equivalent to general relativity, with its usual Lagrangian linear in the scalar curvature. As a consequence of this equivalence, the field equation (46) of the translational gauge theory must also be equivalent to the vacuum Einstein's equation. In fact, through a tedious but straightforward calculation, that equation can be reduced to

$$
h\left(\stackrel{\circ}{R}_{\rho \theta}-\frac{1}{2} g_{\rho \theta} \stackrel{\circ}{R}\right)=0 .
$$

\section{GRAVITATION AS A MANIFESTATION OF TORSION: FORCE EQUATION}

Let us return to the gravitational force equation (19), which assumes now the form

$$
h^{\alpha}{ }_{\mu} \frac{d u_{\alpha}}{d s}=c^{-2} F^{\alpha}{ }_{\mu \nu} u_{\alpha} u^{\nu} .
$$

As we are going to see, there are two different ways of interpreting this force equation. In fact, it can be rewritten alternatively in terms of magnitudes related to the Weitzenböck or to the Riemann spacetime, giving rise, respectively, to the teleparallel and the metric description of gravitation.

We start with the first alternative, which corresponds to transform algebra into tensor indices in such a way to get the force equation (51) written in terms of the Cartan connection only. By using Eq. (25), as well as the relations

$$
h_{\mu}^{\alpha} \frac{d u_{\alpha}}{d s}=\omega_{\mu} \equiv \frac{d u_{\mu}}{d s}-\Gamma_{\theta \mu \nu} u^{\theta} u^{\nu}, \quad u_{\alpha}=h_{\alpha \theta} u^{\theta},
$$

where $\omega_{\mu}$ is the particle four-acceleration in the induced spacetime, it reduces to

$$
\frac{d u_{\mu}}{d s}-\Gamma_{\theta \mu \nu} u^{\theta} u^{\nu}=T_{\theta \mu \nu} u^{\theta} u^{\nu}
$$

The left-hand side of this equation is the Cartan covariant derivative of $u_{\mu}$ along the world line of the particle. The presence of the torsion tensor on its right-hand side, which plays the role of an external force, implies that spinless particles do not follow geodesics in the induced Weitzenböck spacetime. Substituting Eq. (26), it becomes

$$
\frac{d u_{\mu}}{d s}-\Gamma_{\theta \nu \mu} u^{\theta} u^{\nu}=0
$$

Notice that, as $\Gamma_{\theta \nu \mu}$ is not symmetric in the last two indices, this is in fact not a geodesic equation. Actually, it is a force equation describing the interaction of a spinless particle with the gravitational field. According to this description, the only effect of the gravitational field is to induce a torsion in spacetime, which will then be responsible for determining the trajectory of the particle.

\section{GRAVITATION AS A MANIFESTATION OF CURVATURE: GEODESIC EQUATION}

Again, we transform algebra into spacetime indices, but now in such a way to get the force equation (51) written in terms of the Levi-Cività connection only. Following the same steps used earlier, we get

$$
\frac{d u_{\mu}}{d s}-\Gamma_{\theta \mu \nu} u^{\theta} u^{\nu}=T_{\theta \mu \nu} u^{\theta} u^{\nu}
$$

Then, by taking into account the symmetry of $u^{\theta} u^{\nu}$ under the exchange $(\theta \leftrightarrow \nu)$, we can rewrite it as

$$
\frac{d u_{\mu}}{d s}-\Gamma_{\theta \mu \nu} u^{\theta} u^{\nu}=K_{\mu \theta \nu} u^{\theta} u^{\nu} .
$$

Noticing that $K_{\mu \theta \nu}$ is skew symmetric in the first two indices, and using Eq. (35) to express $\left(K_{\theta \mu \nu}-\Gamma_{\theta \mu \nu}\right)$, Eq. (56) becomes

$$
\frac{d u_{\mu}}{d s}-\stackrel{\circ}{\Gamma}_{\theta \mu \nu} u^{\theta} u^{\nu}=0
$$

This is precisely the geodesic equation of general relativity, which means that the trajectories followed by spinless particles are geodesics of the induced Riemann spacetime. According to this description, therefore, the only effect of the gravitational field is to induce a curvature in spacetime, which will then be responsible for determining the trajectory of the particle.

\section{CONCLUSIONS}

In the context of a gauge theory for the translation group, we have succeeded in obtaining a gravitational analogue of 
the Lorentz force equation. This force equation determines the trajectory to be followed by a spinless particle submitted to a gauge gravitational field, in a flat Minkowski spacetime. According to this approach, the trajectory of the particle is described in the very same way the Lorentz force describes the trajectory of a charged particle in the presence of an electromagnetic field. This force equation, however, can be rewritten in terms of magnitudes related to either the teleparallel or the Riemannian structures induced in spacetime by the presence of gravitation, which is represented by a nontrivial tetrad field.

In the first alternative we consider the zero-curvature Cartan connection $\Gamma_{\theta \nu \mu}$ defined on spacetime. Its torsion will be the only manifestation of the gravitational field in this case. In terms of this connection, the force equation (19) becomes

$$
\frac{d u_{\mu}}{d s}-\Gamma_{\theta \nu \mu} u^{\theta} u^{\nu}=0
$$

It is important to remark that, as $\Gamma_{\theta \nu \mu}$ is not symmetric in the last two indices, this is not a geodesic equation, which means that the trajectories followed by spinless particles are not geodesics of the induced Weitzenböck spacetime. In a locally inertial coordinate system the Cartan connection $\Gamma_{\theta \nu \mu}$ becomes skew symmetric in the first two indices, which is the teleparallel version of the normal coordinate condition $\partial_{\mu} g_{\theta \nu}=0$ of general relativity. In this coordinate system, therefore, owing to the symmetry of $u^{\theta} u^{\nu}$, the force equation (58) becomes the equation of motion of a free particle. This is the teleparallel version of the (strong) equivalence principle.

In the second alternative we consider the torsionless LeviCività connection $\stackrel{\circ}{\Gamma}_{\theta \mu \nu}$ defined on spacetime. Its curvature will be the only manifestation of the gravitational field now. In this case, the force equation (19) is reduced to

$$
\frac{d u_{\mu}}{d s}-\stackrel{\circ}{\Gamma}_{\theta \mu \nu} u^{\theta} u^{\nu}=0
$$

This is the geodesic equation of general relativity, which is an equation written in the underlying Riemann spacetime. It corresponds mathematically to the vanishing of the LeviCività covariant derivative of $u_{\mu}$ along the world line of the particle. In a locally inertial coordinate system, the first derivative of the metric tensor vanishes, the Levi-Cività connection vanishes as well, and the geodesic equation (59) becomes the equation of motion of a free particle. This is the usual version of the (strong) equivalence principle as formulated in the general theory of relativity [17].

Notice the difference in the index contractions between the connections and the four-velocities in Eqs. (58) and (59). This difference is responsible for the different characters of these equations: The first is a force equation written in the underlying Weitzenböck spacetime, and the second is a true geodesic equation written in the induced Riemann spacetime.
Furthermore, as can be easily verified, both equations yield, for velocities sufficiently small, the usual Newtonian limit [17]

$$
\phi \equiv-B_{00}=-\frac{G M}{r},
$$

with $\phi$ the Newton gravitational potential.

It is interesting to remark that any one of Eqs. (58) and (59) can be deduced from a variational principle with the action

$$
S=-\int_{a}^{b} m c d s
$$

where $d s=\left(g_{\mu \nu} d x^{\mu} d x^{\nu}\right)^{1 / 2}$ is the spacetime interval and $g_{\mu \nu}=\eta_{\alpha \beta} h^{\alpha}{ }_{\mu} h^{\beta}{ }_{\nu}$. In contrast to the Minkowskian action (17), the interaction of the particle with the gravitational field is in this case given by the presence of the metric tensor $g_{\mu \nu}$ in $d s$ or, alternatively, in $u^{2}=g_{\mu \nu} u^{\mu} u^{\nu}$ if one opts for using a Lagrangian formalism.

Now, as both Eqs. (58) and (59) are deduced from the same force equation (19), they must be equivalent ways of describing the same physical trajectory. In fact, it is easy to see that any one of them can be obtained from the other by substituting the relation

$$
\Gamma_{\theta \mu \nu}=\stackrel{\circ}{\Gamma}_{\theta \mu \nu}+K_{\theta \mu \nu}
$$

In general relativity, the presence of a gravitational field is expressed by a torsionless metric connection, whose curvature determines the intensity of the gravitational field and, consequently, the trajectories to be followed by spinless particles under the influence of the gravitational field. On the other hand, in the teleparallel description of gravitation, the presence of a gravitational field is expressed by a flat Cartan connection, whose torsion is now the entity responsible for determining the intensity of the gravitational field and, consequently, the trajectories to be followed by spinless particles under the influence of the gravitational field. Thus, we can say that the gravitational interaction can be described either in terms of the curvature of spacetime, as is usually done in general relativity, or in terms of the torsion of spacetime. Both interpretations result in being completely equivalent in the sense that they give the same physical trajectory for a spinless particle in a gravitational field. Whether gravitation requires a curved or a torsioned spacetime, therefore, turns out to be a matter of convention. Moreover, contrary to the old belief [20] that only particles with spin could detect the teleparallel geometry, scalar matter being able to feel the metric geometry only, our results imply that scalar matter is able to feel any one of these geometries.

\section{ACKNOWLEDGMENTS}

The authors would like to thank R. Aldrovandi for useful discussions and for a critical reading of the manuscript. They would also like to thank FAPESP-Brazil and CNPq-Brazil, for financial support. 
[1] C. Møller, K. Dan. Vidensk. Selsk. Mat. Fys. Skr. 1, No. 10 (1961).

[2] C. Pellegrini and J. Plebanski, K. Dan. Vidensk. Selsk. Mat. Fys. Skr. 2, No. 2 (1962).

[3] K. Hayashi and T. Nakano, Prog. Theor. Phys. 38, 491 (1967).

[4] K. Hayashi and T. Shirafuji, Phys. Rev. D 19, 3524 (1979).

[5] F. W. Hehl, in Cosmology and Gravitation, edited by P. G. Bergmann and V. de Sabbata (Plenum, New York, 1980).

[6] W. Kopczyński, J. Phys. A 15, 493 (1982).

[7] R. de Azeredo Campos and C. G. Oliveira, Nuovo Cimento B 74, 83 (1983).

[8] F. Müller-Hissen and J. Nitsch, Gen. Relativ. Gravit. 17, 747 (1985).

[9] E. W. Mielke, Ann. Phys. (N.Y.) 219, 78 (1992).

[10] F. W. Hehl J. D. McCrea, E. W. Mielke, and Y. Ne'eman, Phys. Rep. 258, 1 (1995).
[11] R. Weitzenböck, Invariantentheorie (Noordhoff, Gronningen, 1923).

[12] R. Aldrovandi and J. G. Pereira, An Introduction to Geometrical Physics (World Scientific, Singapore, 1995).

[13] J. W. Maluf, J. Math. Phys. 35, 335 (1994).

[14] L. D. Landau and E. M. Lifshitz, The Classical Theory of Fields (Pergamon, Oxford, 1971).

[15] S. K. Wong, Nuovo Cimento A 65, 689 (1970).

[16] W. Drechsler, Phys. Lett. 90B, 258 (1980).

[17] See, for example, S. Weinberg, Gravitation and Cosmology (Wiley, New York, 1972).

[18] T. W. B. Kibble, J. Math. Phys. 2, 212 (1961).

[19] See, for example, P. Ramond, Field Theory: a Modern Primer (Benjamin/Cummings, Reading, MA, 1981).

[20] J. Nitsch and F. W. Hehl, Phys. Lett. 90B, 98 (1980). 